\title{
Effects of flow reduction and spillways on the composition and structure of benthic macroinvertebrate communities in a Brazilian river reach
}

\author{
Maroneze, DM., Tupinambás, TH., França, JS. and Callisto, M.* \\ Laboratório de Ecologia de Bentos, Departamento de Biologia Geral, Instituto de Ciências Biológicas, \\ Universidade Federal de Minas Gerais - UFMG, Av. Antônio Carlos, 6627, Pampulha, \\ CP 486, CEP 30161-970, Belo Horizonte, MG, Brazil \\ *e-mail: callistom@ufmg.br \\ Received July 16, 2010 - Accepted September 1, 2010 - Distibuted August 31, 2011
}

(With 2 figures)

\begin{abstract}
Dams are a major threat to aquatic biological diversity. By altering the natural flow of rivers, dams modify fluvial habitats, making them unsuitable for the growth and reproduction of many aquatic species. The aim of this study was to evaluate the effects of a reduced flow reach (RFR) on benthic macroinvertebrate communities. Benthic macroinvertebrates were collected at six sites downstream of the Amador Aguiar Power Plant I before (lotic phase) and after (semi-lentic phase) Araguari River mean flow was reduced from 346 to $7 \mathrm{~m}^{3} . \mathrm{s}^{-1}$. Changes in macroinvertebrates richness, diversity and total biomass were not observed. Ablabesmyia, Tanytarsus (Chironomidae, Diptera), Leptoceridae and Polycentropodidae (Trichoptera) densities significantly increased the first year after flow reduction and the construction of spillways ( $t$-test; $\mathrm{p}<0.05)$. An analysis of similarity (ANOSIM) showed statistical differences in taxonomical composition despite considerable overlap in communities between the lotic and semi-lentic phases $(\mathrm{R}=0.3 ; \mathrm{p}<0.01)$. In both phases, the macroinvertebrates were characterised by the dominance of groups tolerant to human disturbance (e.g., Chironomidae, Ceratopogonidae and Oligochaeta) and by the presence of the alien bivalve species Corbicula fluminea (Veneroidae), suggesting that the river was already degraded before the hydraulic modifications. Since the 1980s, the Araguari River has been continuously subjected to human pressures (e.g., cascade dams, urbanization and replacement of native vegetation by pasture and crops). These activities have led to impoverishment of biological communities and have consequently altered the ecosystem.
\end{abstract}

Keywords: spillways, reduced flow, dams, benthic, anthrophic impacts.

\section{Efeitos da redução de vazão e construção de soleiras vertentes sobre a composição e estrutura de comunidades de macroinvertebrados bentônicos em um trecho de rio brasileiro}

\begin{abstract}
Resumo
As represas constituem uma das principais ameaças à diversidade biológica. Ao alterarem a vazão natural de um rio, modificam os habitats fluviais tornando-os inadequados para o crescimento e reprodução de diversas espécies aquáticas. $\mathrm{O}$ objetivo deste estudo foi avaliar os efeitos de um trecho de vazão reduzida sobre as comunidades de macroinvertebrados bentônicos. Os organismos foram coletados em seis estações amostrais à jusante da UHE Amador Aguiar I antes (fase lótica) e após (fase semilêntica) a vazão média do rio Araguari (MG) ter sido reduzida de 346 para $7 \mathrm{~m}^{3} . \mathrm{s}^{-1}$. Não foram registradas mudanças na riqueza, diversidade e biomassa total dos macroinvertebrados. As densidades (ind. $\mathrm{m}^{-2}$ ) de Ablabesmyia, Tanytarsus (Diptera), Leptoceridae e Polycentropodidae (Trichoptera) aumentaram significativamente no primeiro ano após a redução da vazão e construção das soleiras vertentes $(t$-test; $\mathrm{p}<0,05)$. Uma análise de similaridade (ANOSIM) apontou diferenças significativas na composição taxonômica, porém houve sobreposição das comunidades entre as fases lótica e semilêntica $(\mathrm{R}=0,3 ; \mathrm{p}<0,01)$. Em ambas as fases a macrofauna caracterizou-se pela dominância de grupos tolerantes a distúrbios antrópicos (p. ex. Chironomidae, Ceratopogonidae e Oligochaeta) e pela presença do bivalve invasor Corbicula fluminea, sugerindo que o rio já estava degradado antes das modificações hidráulicas. Desde a década de 1980 o rio Araguari tem sido continuamente submetido a impactos antrópicos (barramentos em cascata, urbanização e a substituição da vegetação por pastagens e cultivos agrícolas). Essas atividades têm levado ao empobrecimento das comunidades biológicas e, consequentemente, alterado o funcionamento do ecossistema.
\end{abstract}

Palavras-chave: soleiras vertentes, vazão reduzida, represas, bentos, impactos antrópicos. 


\section{Introduction}

The construction of reservoirs is one of the oldest forms of human intervention in aquatic ecosystems (Tundisi and Matsumura-Tundisi, 2008). Currently, all continents but Antarctica have dams in their major basins (Nilsson et al., 2005). In Brazil, there are over 600 dams, which were constructed mainly for hydropower (Agostinho et al., 2005). Although they aid social and economic development (Tundisi et al., 2008), dams alter natural flow regimes (Agostinho et al., 2008), including production of reaches with residual flow that reduce water quality and disturb biological communities (Cortes et al., 2002).

Benthic macroinvertebrates are one of the aquatic communities most affected by reduced flow (Dewson et al., 2007a). Low and constant flows alter benthic communities by altering sediment texture, temperature, and dissolved oxygen (Cortes et al., 2002). The availability of habitats for the growth and foraging of many species is also altered (James et al., 2007), especially for taxa with morphological adaptations (e.g., dorsally flattened body) to better resist high velocities, which include Ephemeroptera and Plecoptera (Merritt and Cummins, 1996).

By changing the current velocity and limiting the species distribution to residual pools, reduced flow accentuates predation and competition (Dewson et al., 2007b). As the water volume decreases, the local density of predators may increase, forcing prey to escape through drift ((Dewson et al., 2007a). During laboratory tests, it was confirmed that larvae of Simuliidae (Simulium vitattum) are easily captured by flatworms (Dugesia dorotocephala) in sites where the water speed is low (Hansen et al., 1991). Predation impacts are altered if habitats with high current velocities are present where preys are less accessible to potential predators (Malmqvist and Sackmann, 1996). Hydropsychidae larvae live clustered in rapids where their filtration feeding habit is favored (Georgian and Thorp, 1992). When the water volume decreases these larvae can move to areas where flows are greater, increasing the competition for space and food resources (Dewson et al., 2007a).

In river reaches with reduced flows, it is common to detect declines in density, diversity through the disappearance of taxa susceptible to flow regime changes (McIntosh et al., 2002; Kinzie et al., 2006). Disturbances may consequently affect ecological processes. The ability of macroinvertebrates to convert plant and microbial food bases into animal tissue is a vital process in aquatic food webs, and loss of macroinvertebrates community structure (e.g., elimination of gatherer or shredder populations) can alter the supply of matter and energy for the entire ecosystem (Graça, 2001).

A strategy employed in some European countries to mitigate the negative effects of flow reduction on aquatic communities (mainly fishes and macroinvertebrates) is the construction of small dams in regulated reaches (Cortes et al., 2002). These structures, called spillways (soleiras vertentes in Brazil, CCBE (2007)), may benefit the biota by forming backwaters that maintain a continuous water surface in the river (Brittain and L'Abée-Lund,
1995). However, the spillways should be constructed in small numbers and interspersed by rapids; otherwise, they could degrade the environment, retain large quantities of debris, and prevent the drift of aquatic macroinvertebrates (Fjellheim and Raddum, 1996).

The aim of this study was to assess the taxonomic composition, structure and biomass of benthic macroinvertebrate communities in a reach with reduced flow before and after spillway (RFR) construction. In Brazil, the effects of small dams on the benthic community have received little attention and were recently examined by Almeida et al. (2009). These authors compared upstream sites (references) with those downstream the impoundments (impaired) in two third order streams of Rio de Janeiro whereas the present research evaluated changes in a large river reach prior and after its damming. Together with the study above, this represents one of the first Brazilian ecological approaches to assess macroinvertebrate community responses to an artificially reduced and constant flow, as well as the spillways construction. This is an important study considering the susceptibility of benthos to habitat changes (Ogbeibu and Oribhabor, 2002), their role as bioindicators of the impacts caused by altered flow regimes (Dewson et al., 2007a), and the increased number of dewatered rivers in Brazil.

The hypothesis tested was that conversion of a lotic habitat into a semi-lentic habitat resulting from reduced flow and construction of spillways would alter the composition and structure of the macroinvertebrate communities. Under lotic conditions, it was expected to find higher taxa richness and a predominance of collector-filter organisms, which obtain food by filtering fine organic particles transported by the water flow. Under semi-lentic conditions, it was expected to find an increase in the abundance of taxonomic groups associated with calm water and a higher proportion of collector-gatherer organisms resulting from the accumulation of fine organic matter in the sediment and reduced current velocities.

\subsection{Study area}

The study was carried out in a reach of the Araguari River, with residual flow located downstream of Amador Aguiar I Reservoir (MG), between $18^{\circ} 20^{\prime} \mathrm{S}$ and $46^{\circ} 00^{\prime} \mathrm{W}$ and $20^{\circ} 10^{\prime} \mathrm{S}$ and $48^{\circ} 50^{\prime} \mathrm{W}$ (Figure 1). This $9 \mathrm{~km}$ long reach is $90 \mathrm{~m}$ wide and drains areas of sandstone and basalt; the valley bottom is in weathered granite and gneiss (Rodrigues, 2002). The average annual rainfall is $1,555 \mathrm{~mm} /$ year with a rainy season from October to March and a dry season from May to September. The mean annual temperature is $22{ }^{\circ} \mathrm{C}$ (Rosa et al., 2006). Pasture is the dominant vegetation around the reduced flow reach, representing $39 \%$ of the basin. The remaining land uses are preserved Cerrado (17.8\%), annual crops (17.5\%), temporary crops $(15.4 \%)$, urban areas $(7.5 \%)$ and others (2.1\%) (J.F. Silva, pers. com).

After the Amador Aguiar I Reservoir was filled (Table 1), the mean flow of the downstream reach was reduced from 346 to $7 \mathrm{~m}^{3} \cdot \mathrm{s}^{-1}$ (the minimum value recorded for the river 


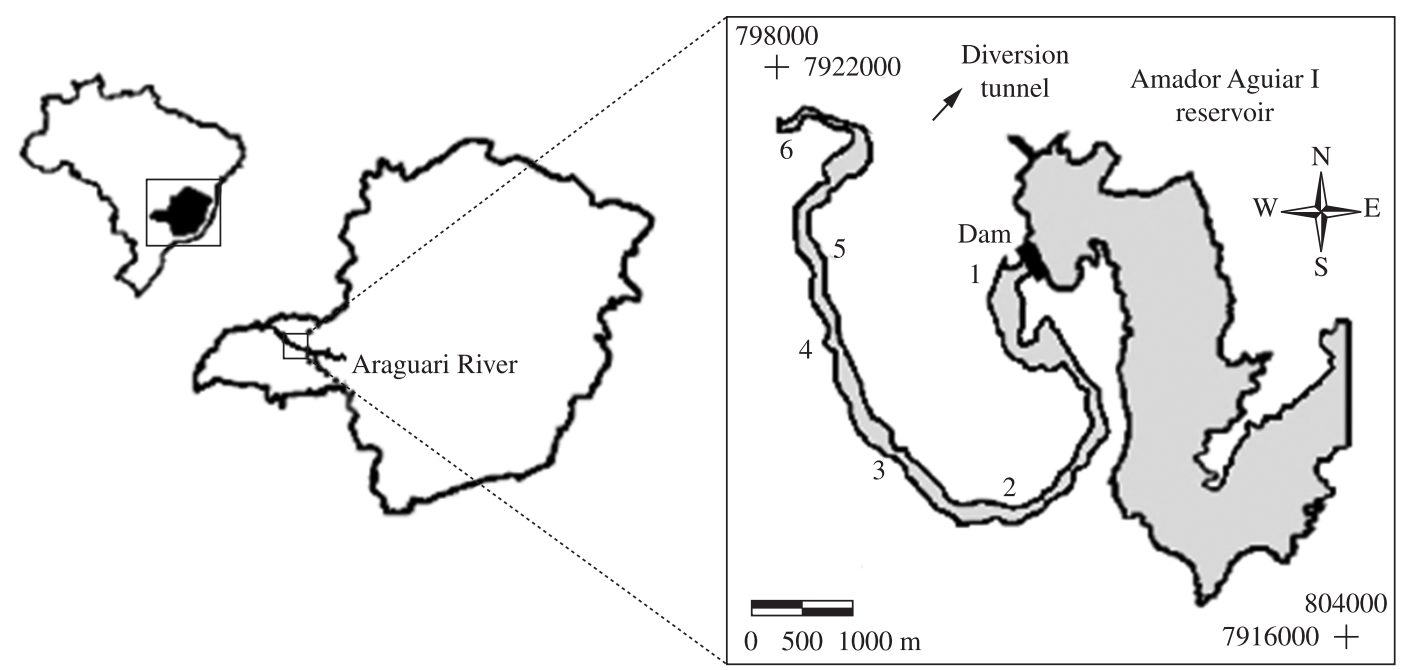

Figure 1. Study area showing the sampling stations (Minas Gerais, Brazil).

Table 1. Technical, morphometric and limnological characteristics of the Amador Aguiar I Reservoir (Minas Gerais, Brazil).

\begin{tabular}{lc}
\hline \multicolumn{1}{c}{ Characteristics* } & Amador Aguiar I reservoir \\
\hline River diversion & July 2004 \\
Filling period & December 2005 \\
Start of reservoir operation and reduction in downstream flow & May 2006 \\
Flooded terrestrial area $\left(\mathrm{km}^{2}\right)$ & 18.66 \\
Volume of water $\left(\right.$ million $\left.{ }^{3}\right)$ & 241 \\
Reservoir depth at the dam $(\mathrm{m})$ & 52 \\
Length of the dam $(\mathrm{m})$ & 610 \\
Length of the reservoir $(\mathrm{km})$ & 75 \\
Installed capacity $(\mathrm{MW})$ & 240 \\
Trophic status & Ultraoligotrophic \\
Chlorophyll-a $\left(\mathrm{mg} . \mathrm{L}^{-1}\right)$ & $2.14-4.28^{+}$ \\
Electrical conductivity $\left(\mu \mathrm{S} \mathrm{cm}^{-1}\right)$ & $30-32^{+}$ \\
pH & $5.8-6.2^{+}$ \\
Total alkalinity $\left(\mathrm{meq} . \mathrm{L}^{-1} \mathrm{CO}_{2}\right)$ & 0.19 \\
Dissolved oxygen $\left(\mathrm{mg} . \mathrm{L}^{-1}\right)$ & $6.0-7.2^{+}$ \\
Total phosphorus concentration $\left(\mu \mathrm{g} . \mathrm{L}^{-1}\right)$ & $8.10-11.78^{+}$ \\
Total nitrogen concentration $\left(\mu \mathrm{g} . \mathrm{L}^{-1}\right)$ & $276.95-355.20^{+}$ \\
\hline
\end{tabular}

*Data according to CCBE (2007) and Valadares (2007). ${ }^{+}$(Minimum - Maximum value).

in the 10 years preceding plant construction). To reestablish the water level, five small dams (spillways) were built in the reduced flow reach. The spillways are 3 to $10 \mathrm{~m}$ high and produced a series of semi lentic reservoirs.

\section{Methods}

This study was conducted in two stages: the first (lotic phase) was characterised by natural reach conditions, with well-developed riffles and pools free from the reservoir and spillway influences. The second stage (semi-lentic phase) occurred after dam construction when the reach was altered by reduced flow and fragmented by spillways.
The predominant aquatic habitats under this condition were composed of large backwaters and pools.

Six sites were random sampled along the reduced flow reach averaging $1.5 \mathrm{~km}$ apart (Table 2; Figure 1). All of them were visited four times: once during the lotic phase in both June and October 2005 and once during the semilentic phase in both June and December 2008 (the first year after spillways). Thus, for each phase studied (lotic and semi-lentic) data were collected in dry and rainy seasons, representing a complete seasonal cycle. The total rainfall was $5 \mathrm{~mm}$ in July and $130 \mathrm{~mm}$ in October 2005, and $5 \mathrm{~mm}$ in June and $260 \mathrm{~mm}$ in December 2008 (Simge, 2010). 
Table 2. Characterisation of sampling stations in the stretch downstream to the Amador Aguiar I Reservoir (Minas Gerais, Brazil) in lotic (2005) and semi-lentic phases (2008).

\begin{tabular}{lcc}
\hline \multicolumn{1}{c}{ Characteristics } & \multicolumn{2}{c}{ Phases } \\
\cline { 2 - 3 } Depth $(\mathrm{m})$ & Lotic & Semi-lentic \\
\hline Width $(\mathrm{m})$ & $2.0-9.0^{+}$ & $1.10-2.10^{+}$ \\
Average flow $\left(\mathrm{m}^{3} \cdot \mathrm{s}^{-1}\right)$ & $50-100^{+}$ & $58-90^{+}$ \\
Predominant habitat type* & 346 & 7 \\
Aquatic plants* & Riffles & Pools \\
Proportion of fine sediment fractions $(<0.25 \mathrm{~mm})(\%)^{* *}$ & Absent & Absent \\
Organic matter content of sediment $(\%)$ & $83-100^{+}$ & $18-96^{+}$ \\
Trophic status & $0.7-5.9^{+}$ & $0.5-16.9^{+}$ \\
\hline
\end{tabular}

*Evaluated according to Callisto et al., (2002a); **Determined according to Callisto and Esteves (1996); ${ }^{+}$Minimum Maximum value).

Water temperature $\left({ }^{\circ} \mathrm{C}\right), \mathrm{pH}$, electrical conductivity $\left(\mu \mathrm{S} . \mathrm{cm}^{-1}\right)$ and turbidity (NTU) were measured in situ with an HORIBA multiprobe (model U-10) and air temperature with a conventional thermometer. Concentrations of dissolved oxygen, total phosphorus and total nitrogen concentrations were determined at the UFMG laboratory according to the Standard Methods for the Examination of Water and Wastewater (Apha, 1992).

At each sampling station, three sediment samples were collected with a van Veen dredge $\left(0.045 \mathrm{~m}^{2}\right)$. Due to the rocky nature of the central axis of the river, the material was only collected along the margins. A total of 72 van Veen sample units were obtained (six sites $x$ three samples/site $\mathrm{x}$ two visits/site $\mathrm{x}$ two phases $=72$ ). The sediment samples were washed through 1.0 and $0.5 \mathrm{~mm}$ sieves and all remaining organisms were identified according to Pérez (1988), Trivinho-Strixino and Strixino (1995), Epler (2001), Costa et al. (2006) and Roque and Trivinho-Strixino (2008). After identification, organisms were dried in a stove at $60{ }^{\circ} \mathrm{C}$ for 48 hours and weighed on a precision scale to determine their biomass $\left(\mathrm{mg} \cdot \mathrm{m}^{-2}\right)$. Mollusks were burned in a muffle furnace at $550{ }^{\circ} \mathrm{C}$ for 4 hours to estimate the weight of mineral parts.

To determine benthic macroinvertebrate community structure, taxonomic richness, Pielou's evenness index, and Shannon-Wiener diversity index (Magurran, 2004) were calculated from the total number of taxa found per van Veen sample, and the densities of organisms were estimated (individuals. $\mathrm{m}^{-2}$ ). Classification into functional feeding groups was based on Merritt and Cummins (1996), Fernández and Dominguez (2001), and Cummins et al. (2005).

\subsection{Data analysis}

Firstly, previous analyses were done to evaluate the significance of differences between the sampling sites and seasons (each phase apart). The values of richness, total density, relative density, total biomass, relative biomass, taxonomic composition and Shannon-Wiener diversity were compared trough ANOVA one-way (sites) ANOSIM and the $t$-test (seasons) $(\alpha=0.05)$. There were no significant differences in these variables. As a consequence, for the following analyses, the variation in community structure among sites or seasons was disregarded.

Changes in limnological characteristics and in the structure of benthic macroinvertebrate communities between the two phases were tested through the $t$-test $(\alpha=0.05)$ using STATISTICA 7.0 software. For these analyses, the three sampling units (van Veen) for each site were pooled, resulting in 12 samples in lotic phase (six sites $\mathrm{X}$ one pooled sample/site $\mathrm{x}$ two visits/site) and 12 samples in semi-lentic phase.

Data were compiled at each site to derive a single value because the three sampling units (van Veen) per site were not independent; this was done to ameliorate the problem of spatial pseudoreplication, recurrent in ecological studies (Hargrove and Pickering, 1992). Each one of the 12 samples per phase was considered one replica. In this study, real replicas (spatial and temporal replication) were virtually impossible to obtain because until now, there is no other regulated river reach in Brazil with similar characteristics (a reach with a constant and artificially reduced flow where benthic macroinvertebrates have been sampled before and after spillway construction). Although statistical inferences should be avoided, impact assessment studies in which replication is undesirable or logically non-existent can be valid and scientifically acceptable; this is particularl y true for field approaches that investigate large scale systems (e.g. catchments and rivers) (Hurlbert, 1984).

The abiotic (electrical conductivity, dissolved oxygen, total phosphorus and total nitrogen) and biotic (density and biomass) variables were $\log$-transformed $(\log \mathrm{x}+1)$ to meet assumptions of normality (Komogorov-Smirnov) and homoscedasticity (Levene) (Zar, 1996). To estimate changes in diversity values between phases, the variances of the Shannon-Wiener index were compared using a $t$-test (Magurran, 2004).

The differences in the taxonomic composition of the benthic macroinvertebrate communities between the lotic and semi-lentic phases were assessed through a non-metric MDS (NMDS) ordination technique, using a Bray-Curtis 
dissimilarity matrix with abundance data transformed $(\log x+1)$ and a Sorensen similarity index with presence/ absence of taxa data. Abundance data favours common species, whereas presence/absence data emphasizes rare species. The NMDS orders all samples in a bidimensional plot, grouping them according to taxonomic similarity (similar samples are close, different samples are further apart).

The statistical significance of the groupings (lotic phase vs. semi-lentic) were tested through similarity analysis (ANOSIM) $(\alpha=0.05)$. The ANOSIM provides a value for interpretation where $\mathrm{R}>0.75$ indicates totally distinct groups, $0.50<\mathrm{R}<0.75$ indicates separated but overlapping groups, $0.25<\mathrm{R}<0.50$ indicates separated but strongly overlapping groups, and $\mathrm{R}<0.25$ represents groups that cannot be distinguished (Clarke and Green, 1988; Michelland et al., 2010). A randomisation process using Monte Carlo testing with 10,000 interactions was conducted to validate the $R$ values observed. A value of $p<0.05$ indicated that the $R$ value observed was not randomly obtained. Analysis of NMDS and ANOSIM were performed with PRIMER 6.0 software.

\section{Results}

\subsection{Physical and chemical variables in the water column}

In both study phases the reach water had near neutral $\mathrm{pH}$; high dissolved oxygen concentrations; and low levels of electric conductivity, turbidity, total-P and total-N (Table 3). Nevertheless, the values for electric conductivity ( $t$-test; $\mathrm{t}=5.71, \mathrm{df}=22, \mathrm{p}<0.01)$, turbidity $(\mathrm{t}=22.97, \mathrm{p}<0.01)$, total-P $(\mathrm{t}=2.23, \mathrm{p}<0.05)$ and total-N $(\mathrm{t}=4.64, \mathrm{p}<0.01)$ were significantly lower in the semi-lentic phase, while temperature was significantly higher $(\mathrm{t}=-2.41, \mathrm{p}<0.05)$. Dissolved oxygen and $\mathrm{pH}$ did not vary significantly between the two phases.

\subsection{Structure and composition of benthic macroinvertebrate communities}

A total of 4,891 benthic macroinvertebrates were found in the 72 sediment samples. The organisms belonged to 50 operational taxonomic units (OTU, 2 Mollusca, 2
Annelida and 46 Arthropoda), 36 OTU were collected in the lotic phase and 45 in the semi-lentic phase. Taxonomic richness did not differ significantly between the two phases (Figure 2a) nor did Shannon-Wiener diversity (Table 4). Chironomidae was the dominant group, accounting for over $69 \%$ of the individuals (Figure 2f), with Polypedilum (18.2\%) and Aedokritus (16.5\%) the dominant genera in the lotic phase and Tanytarsus (23.2\%) and Aedokritus $(16.4 \%)$ in the semi-lentic phase (Table 4). Groups (no Chironomidae) with relative abundances greater than $2.5 \%$ were Oligochaeta (14.4 and 6.4\%, lotic and semi-lentic phase, respectively), Ceratopogonidae (8.0 and 2.8\%) and the alien bivalve Corbicula fluminea (Müller, 1774) (4.2 and $3.5 \%$ ).

The organisms were grouped into five feeding guilds: collector-gatherers, collector-filterers, predators, scrapers, and shredders. During the lotic phase, collector-gatherers $(53.7 \%)$ predominated numerically, but collector-filterers $(38.9 \%)$ predominated in the semi-lentic phase. Shredders were proportionately more abundant during the lotic phase than the semi-lentic (18.2 vs. $6.5 \%)$, while scrapers were virtually absents $(<0.2 \%)$ (Figure $2 \mathrm{~d})$.

Total density was significantly greater during the semilentic phase $(t$-test; $\mathrm{t}=-2.71, \mathrm{df}=22, \mathrm{p}<0.05)$ (Figure $2 \mathrm{~b}$ ). The OTU with significant density increases were Ablabesmyia $(\mathrm{t}=-3.57, \mathrm{p}<0.01)$, Tanytarsus $(\mathrm{t}=-3.65, \mathrm{p}<0.01)$, Leptoceridae $(\mathrm{t}=-2.27, \mathrm{p}<0.05)$, and Polycentropodidae $(\mathrm{t}=-8.94, \mathrm{p}<0.01)$ (Table 4). Total macroinvertebrates biomass did not differ significantly between the two phases (Figure 2c); however, Ablabesmyia ( $t$-test; $\mathrm{t}=-3.56, \mathrm{df}=22$, $\mathrm{p}<0.01)$, Tanytarsus $(\mathrm{t}=-5.27, \mathrm{p}<0.01)$, Leptoceridae $(\mathrm{t}=-2.20, \mathrm{p}<0.05)$, and Polycentropodidae $(\mathrm{t}=-4.59$, $\mathrm{p}<0.01$ ) had significantly higher biomasses during the semi-lentic phase (Table 4 ). Over $65 \%$ of the total biomass was Corbicula fluminea (Figure 2e).

The NMDS and similarity analyses showed a significant separation between samples collected during the lotic and semi-lentic phases, both for abundance data (ANOSIM; $R=0.32 ; \mathrm{p}<0.01)$ and for presence/absence of taxa $(R=0.30 ; \mathrm{p}<0.01)$. This indicates changes in the taxonomic composition of the benthic macroinvertebrate communities after alteration and fragmentation of the river habitats (Figure $2 \mathrm{~g}, \mathrm{~h}$ ). Of the total OTU recorded, $10 \%$ were

Table 3. Physical and chemical characteristics of water (mean \pm SD) in the dewatered reach downstream of the Amador Aguiar I Reservoir (Minas Gerais, Brazil) in lotic (2005) and semi-lentic phases (2008).

\begin{tabular}{lcc}
\hline \multicolumn{1}{c}{ Abiotic variables } & Lotic phase $(\mathbf{n}=\mathbf{1 2})^{*}$ & Semi-lentic phase $(\mathbf{n}=\mathbf{1 2})$ \\
\hline $\mathrm{pH}$ & $6.87 \pm 0.35$ & $7.20 \pm 0.47$ \\
Electrical conductivity $\left(\mu \mathrm{S} . \mathrm{cm}^{-1}\right)$ & $26.41 \pm 3.76$ & $15.31 \pm 5.57$ \\
Turbidity $(\mathrm{NTU})$ & $11.82 \pm 1.01$ & $1.75 \pm 1.13$ \\
Dissolved oxygen $\left(\mathrm{mg} . \mathrm{L}^{-1}\right)$ & $9.41 \pm 1.25$ & $8.70 \pm 0.37$ \\
Water temperature $\left({ }^{\circ} \mathrm{C}\right)$ & $23.26 \pm 1.60$ & $24.74 \pm 1.37$ \\
Total phosporus concentration $\left(\mu \mathrm{g} . \mathrm{L}^{-1}\right)$ & $18.63 \pm 7.57$ & $12.72 \pm 5.10$ \\
Total nitrogen concentration $\left(\mu \mathrm{g} . \mathrm{L}^{-1}\right)$ & $163.04 \pm 55.42$ & $81.08 \pm 26.93$ \\
\hline
\end{tabular}

* number of samples. 

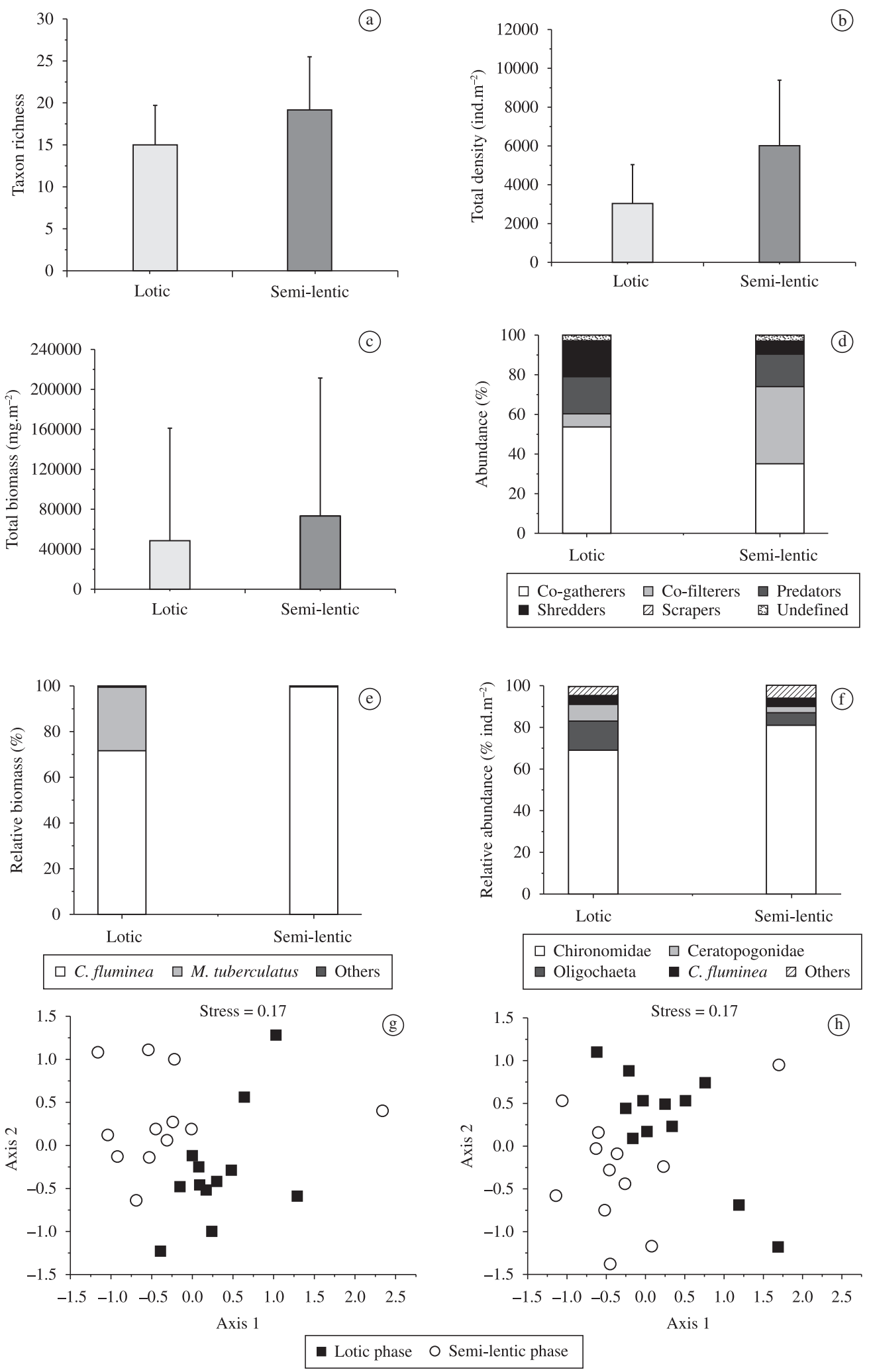

Figure 2. a) OTU richness; b) total density; c) total biomass (mean \pm SD); d) functional feeding group composition \%; e) relative biomass $\%$; f) relative density $\%$; and g) two-dimensional NMDS ordination based on relative abundance - h) presence/absence of benthic macroinvertebrate taxa sampled in the dewatered reach downstream of the Amador Aguiar I Reservoir (Minas Gerais, Brazil) in the lotic (2005) and semi-lentic (2008) phases. 
Table 4. Relative abundance (\% individuals. $\mathrm{m}^{-2}$ ), density (individuals. $\left.\mathrm{m}^{-2}\right)$ and biomass $\left(\mathrm{mg} \cdot \mathrm{m}^{-2}\right)(\mathrm{mean} \pm \mathrm{SD})$ of benthic macroinvertebrate taxa sampled in the reach downstream to the Amador Aguiar I reservoir (Minas Gerais, Brazil) in lotic (2005) and semi-lentic (2008) phases.

\begin{tabular}{|c|c|c|c|c|c|c|c|}
\hline \multirow{3}{*}{ Taxa } & \multirow{3}{*}{ FFG } & \multicolumn{6}{|c|}{ Phases } \\
\hline & & \multicolumn{3}{|c|}{ Lotic $(n=12)$} & \multicolumn{3}{|c|}{ Semi-lentic $(n=12)$} \\
\hline & & $\mathbf{A}$ & D & B & $\mathbf{A}$ & D & B \\
\hline \multicolumn{8}{|l|}{ Mollusca } \\
\hline \multicolumn{8}{|l|}{ Bivalvia } \\
\hline $\begin{array}{l}\text { Corbicula fluminea Müller, } \\
1774\end{array}$ & Co-Fil & $4.2 \pm 7.1$ & $115 \pm 182$ & $\begin{array}{c}34742.1 \pm \\
81328.0\end{array}$ & $3.5 \pm 5.0$ & $170 \pm 243$ & $\begin{array}{l}72941.7 \pm \\
138114.1\end{array}$ \\
\hline \multicolumn{8}{|l|}{ Gastropoda } \\
\hline $\begin{array}{l}\text { Melanoides tuberculatus } \\
\text { Müller, } 1774\end{array}$ & $\mathrm{Co}-\mathrm{Ga}$ & $0.8 \pm 2.8$ & $17 \pm 58$ & $\begin{array}{c}13481.3 \pm \\
46702.2\end{array}$ & $* *$ & $2 \pm 6$ & $1.3 \pm 4.4$ \\
\hline \multicolumn{8}{|l|}{ Annelida } \\
\hline Hirudinea & $\operatorname{Pr}$ & $* *$ & $6 \pm 14$ & $2.3 \pm 3.5$ & - & - & - \\
\hline Oligochaeta & $\mathrm{Co}-\mathrm{Ga}$ & $\begin{array}{c}14.4 \pm \\
12.3\end{array}$ & $387 \pm 316$ & $\begin{array}{l}85.3 \pm \\
122.5\end{array}$ & $6.4 \pm 10.5$ & $369 \pm 664$ & $50.2 \pm 66.4$ \\
\hline \multicolumn{8}{|l|}{ Insecta } \\
\hline \multicolumn{8}{|l|}{ Ephemeroptera } \\
\hline Baetidae & $\mathrm{Co}-\mathrm{Ga}$ & $0.1 \pm 0.3$ & $4 \pm 9$ & $0.1 \pm 0.2$ & $0.2 \pm 0.4$ & $13 \pm 20$ & $1.4 \pm 4.1$ \\
\hline Leptohyphidae & $\mathrm{Co}-\mathrm{Ga}$ & $0.2 \pm 0.6$ & $7 \pm 20$ & $* *$ & - & - & - \\
\hline Leptophlebiidae & $\mathrm{Co}-\mathrm{Ga}$ & $0.1 \pm 0.3$ & $2 \pm 6$ & $* *$ & - & - & - \\
\hline \multicolumn{8}{|l|}{ Odonata } \\
\hline Coenagrionidae & $\operatorname{Pr}$ & - & - & - & $0.1 \pm 0.2$ & $4 \pm 9$ & $3.4 \pm 11.5$ \\
\hline Gomphidae & $\operatorname{Pr}$ & $0.3 \pm 0.4$ & $11 \pm 15$ & $\begin{array}{c}66.1 \pm \\
187.8\end{array}$ & $0.5 \pm 0.5$ & $30 \pm 27$ & $38.2 \pm 87.1$ \\
\hline Libellulidae & $\operatorname{Pr}$ & $0.3 \pm 1.0$ & $4 \pm 9$ & $1.6 \pm 5.6$ & $0.7 \pm 1.8$ & $43 \pm 101$ & $1.6 \pm 2.6$ \\
\hline \multicolumn{8}{|l|}{ Heteroptera } \\
\hline Veliidae & $\operatorname{Pr}$ & - & - & - & $0.1 \pm 0.2$ & $6 \pm 14$ & $0.7 \pm 1.8$ \\
\hline \multicolumn{8}{|l|}{ Coleoptera } \\
\hline Elmidae & $\mathrm{Co}-\mathrm{Ga}$ & $0.1 \pm 0.3$ & $6 \pm 14$ & $1.2 \pm 3.4$ & $* *$ & $2 \pm 6$ & $* *$ \\
\hline \multicolumn{8}{|l|}{ Trichoptera } \\
\hline Hydropsychidae & Co-Fil & - & - & - & $* *$ & $2 \pm 6$ & $0.2 \pm 0.8$ \\
\hline Hydroptilidae & $\mathrm{Co}-\mathrm{Ga}$ & $0.1 \pm 0.4$ & $2 \pm 6$ & $* *$ & $2.5 \pm 7.6$ & $146 \pm 434$ & $4.0 \pm 9.9$ \\
\hline Leptoceridae & $\operatorname{Pr}$ & - & - & - & $0.3 \pm 0.5$ & $22 \pm 46$ & $6.7 \pm 15.8$ \\
\hline Odontoceridae & Scr & - & - & - & $0.1 \pm 0.3$ & $7 \pm 14$ & $0.1 \pm 2.2$ \\
\hline Polycentropodidae & Co-Fil & - & - & - & $1.8 \pm 2.0$ & $102 \pm 116$ & $11.5 \pm 14.0$ \\
\hline \multicolumn{8}{|l|}{ Diptera } \\
\hline Ceratopogonidae & $\operatorname{Pr}$ & $8.0 \pm 6.3$ & $239 \pm 235$ & $12.3 \pm 9.4$ & $2.8 \pm 2.3$ & $150 \pm 136$ & $8.3 \pm 7.7$ \\
\hline \multicolumn{8}{|l|}{ Chironomidae } \\
\hline \multicolumn{8}{|l|}{ Tanypodinae } \\
\hline $\begin{array}{l}\text { Ablabesmyia Johhansen, } \\
1905\end{array}$ & $\operatorname{Pr}$ & $0.5 \pm 0.7$ & $19 \pm 39$ & $4.0 \pm 12.1$ & $3.3 \pm 3.6$ & $215 \pm 287$ & $9.2 \pm 7.0$ \\
\hline Coelotanypus Kieffer, 1913 & $\operatorname{Pr}$ & - & - & - & $0.1 \pm 0.2$ & $6 \pm 14$ & $1.1 \pm 3.9$ \\
\hline Djalmabatista Fittkau, 1908 & $\operatorname{Pr}$ & $2.5 \pm 3.2$ & $63 \pm 63$ & $3.1 \pm 4.2$ & $2.4 \pm 4.8$ & $185 \pm 450$ & $6.4 \pm 13.1$ \\
\hline $\begin{array}{l}\text { Fittkauimyia Karunakaran, } \\
1969\end{array}$ & $\operatorname{Pr}$ & - & - & - & $0.5 \pm 1.2$ & $30 \pm 64$ & $2.9 \pm 6.3$ \\
\hline Labrundinia Fittkau, 1962 & $\operatorname{Pr}$ & - & - & - & $0.4 \pm 1.3$ & $20 \pm 64$ & $0.2 \pm 0.8$ \\
\hline
\end{tabular}

$\mathrm{A}=$ abundance, $\mathrm{D}=$ density, $\mathrm{B}=$ biomass. FFG = Functional Feeding Groups, Co-Ga = collector-gatherers, Co-Fil = collector-filterers, $\operatorname{Pr}=$ predators, $\mathrm{Shr}=$ shredders, $\mathrm{Scr}=$ scrapers. $(*)=$ undefined, $(* *)=$ value $<0.1,(-)=$ absent. 
Table 4.. Continued...

\begin{tabular}{|c|c|c|c|c|c|c|c|}
\hline \multirow{3}{*}{ Taxa } & \multirow{3}{*}{ FFG } & \multicolumn{6}{|c|}{ Phases } \\
\hline & & \multicolumn{3}{|c|}{ Lotic $(n=12)$} & \multicolumn{3}{|c|}{ Semi-lentic $(n=12)$} \\
\hline & & $\mathbf{A}$ & D & B & $\mathbf{A}$ & D & B \\
\hline Pentaneura Phillipi, 1865 & $\operatorname{Pr}$ & - & - & - & $* *$ & $2 \pm 6$ & $* *$ \\
\hline Tanypus Meigen, 1803 & $\operatorname{Pr}$ & $0.1 \pm 0.2$ & $2 \pm 6$ & $0.2 \pm 0.6$ & $7.2 \pm 22.2$ & $115 \pm 270$ & $12.9 \pm 32.9$ \\
\hline \multicolumn{8}{|l|}{ Chironominae } \\
\hline Aedokritus Roback, 1958 & $\mathrm{Co}-\mathrm{Ga}$ & $\begin{array}{c}16.5 \pm \\
18.7\end{array}$ & $\begin{array}{c}637 \pm \\
1001\end{array}$ & $\begin{array}{c}56.9 \pm \\
93.0\end{array}$ & $\begin{array}{c}16.4 \pm \\
22.5\end{array}$ & $\begin{array}{l}943 \pm \\
1253\end{array}$ & $73.0 \pm 93.8$ \\
\hline Chironomus Meigen, 1803 & $\mathrm{Co}-\mathrm{Ga}$ & $1.1 \pm 1.4$ & $35 \pm 47$ & $3.6 \pm 7.7$ & $1.5 \pm 3.8$ & $93 \pm 198$ & $25.6 \pm 62.3$ \\
\hline Cladopelma Kieffer, 1921 & $\mathrm{Co}-\mathrm{Ga}$ & $6.8 \pm 5.4$ & $265 \pm 293$ & $5.4 \pm 7.0$ & $3.5 \pm 4.8$ & $211 \pm 273$ & $4.3 \pm 4.7$ \\
\hline $\begin{array}{l}\text { Cryptochironomus Kieffer, } \\
1918\end{array}$ & $\operatorname{Pr}$ & $7.5 \pm 5.8$ & $211 \pm 150$ & $8.2 \pm 6.2$ & $2.2 \pm 1.8$ & $135 \pm 115$ & $8.8 \pm 7.8$ \\
\hline $\begin{array}{l}\text { Demicryptochironomus } \\
\text { Lenz, } 1941\end{array}$ & $\mathrm{Co}-\mathrm{Ga}$ & $1.0 \pm 1.7$ & $35 \pm 48$ & $2.1 \pm 3.0$ & $0.2 \pm 0.4$ & $13 \pm 38$ & $0.4 \pm 1.2$ \\
\hline Dicrotendipes Kieffer, 1913 & $\mathrm{Co}-\mathrm{Ga}$ & - & - & - & $0.4 \pm 0.9$ & $19 \pm 40$ & $1.9 \pm 4.8$ \\
\hline $\begin{array}{l}\text { Endotribelos Grodhaus, } \\
1987\end{array}$ & Co-Ga & $0.1 \pm 0.4$ & $4 \pm 13$ & $* *$ & $0.2 \pm 0.4$ & $11 \pm 26$ & $0.1 \pm 0.4$ \\
\hline $\begin{array}{l}\text { Fissimentum Cranston \& } \\
\text { Nolte, } 1916\end{array}$ & $*$ & $2.4 \pm 3.2$ & $67 \pm 123$ & $2.8 \pm 5.7$ & $2.1 \pm 2.7$ & $135 \pm 256$ & $8.5 \pm 11.8$ \\
\hline $\begin{array}{l}\text { Goeldichironomus Fittkau, } \\
1965\end{array}$ & $\mathrm{Co}-\mathrm{Ga}$ & $* *$ & $2 \pm 6$ & $0.2 \pm 0.7$ & $0.1 \pm 0.2$ & $6 \pm 10$ & $1.3 \pm 2.9$ \\
\hline Pelomus Reiss, 1989 & $\mathrm{Co}-\mathrm{Ga}$ & $5.3 \pm 5.0$ & $211 \pm 291$ & $5.7 \pm 5.9$ & $0.6 \pm 0.1$ & $41 \pm 75$ & $0.3 \pm 0.6$ \\
\hline Nilothauma Kieffer, 1921 & $\mathrm{Co}-\mathrm{Ga}$ & $0.1 \pm 0.3$ & $3 \pm 14$ & $0.2 \pm 0.5$ & $0.4 \pm 0.5$ & $33 \pm 60$ & $1.0 \pm 1.5$ \\
\hline $\begin{array}{l}\text { Paralauterboniella Lenz, } \\
1941\end{array}$ & $*$ & $0.5 \pm 0.8$ & $20 \pm 32$ & $0.7 \pm 1.3$ & $0.7 \pm 0.7$ & $43 \pm 45$ & $0.5 \pm 0.9$ \\
\hline Polypedilum Kieffer, 1913 & Sch & $\begin{array}{c}18.2 \pm \\
17.2\end{array}$ & $543 \pm 637$ & $\begin{array}{c}12.0 \pm \\
21.0\end{array}$ & $3.5 \pm 3.3$ & $257 \pm 354$ & $6.2 \pm 8.9$ \\
\hline $\begin{array}{l}\text { Pseudochironomus } \\
\text { Mallock, } 1915\end{array}$ & Co-Ga & $0.1 \pm 0.3$ & $4 \pm 9$ & $0.1 \pm 0.3$ & $0.8 \pm 2.2$ & $70 \pm 209$ & $3.9 \pm 9.6$ \\
\hline $\begin{array}{l}\text { Stempellina Thienemann \& } \\
\text { Bause, } 1913\end{array}$ & $\mathrm{Co}-\mathrm{Ga}$ & $0.1 \pm 0.3$ & $4 \pm 9$ & $0.1 \pm 0.4$ & $0.4 \pm 0.8$ & $26 \pm 45$ & $0.6 \pm 0.9$ \\
\hline $\begin{array}{l}\text { Stenochironomus Kieffer, } \\
1919\end{array}$ & Sch & - & - & - & $0.1 \pm 0.3$ & $7 \pm 20$ & $0.6 \pm 1.5$ \\
\hline $\begin{array}{l}\text { Tanytarsus van der Wulp, } \\
1984\end{array}$ & Co-Fil & $4.0 \pm 4.8$ & $89 \pm 81$ & $1.8 \pm 2.9$ & $\begin{array}{c}23.2 \pm \\
19.2\end{array}$ & $\begin{array}{l}2072 \pm \\
2585\end{array}$ & $35.2 \pm 40.5$ \\
\hline Zavreliella Kieffer, 1920 & Co-Ga & $0.1 \pm 0.3$ & $6 \pm 14$ & $0.1 \pm 0.4$ & $0.6 \pm 1.3$ & $39 \pm 82$ & $0.5 \pm 1.5$ \\
\hline \multicolumn{8}{|l|}{ Orthocladiinae } \\
\hline $\begin{array}{l}\text { Cricotopus van der Wulp, } \\
1874\end{array}$ & Sch & $1.0 \pm 2.3$ & $17 \pm 38$ & $0.6 \pm 1.1$ & $2.0 \pm 1.4$ & $130 \pm 240$ & $3.1 \pm 6.4$ \\
\hline $\begin{array}{l}\text { Psectrocladius Kieffer, } \\
1906\end{array}$ & Co-Ga & $0.8 \pm 1.4$ & $22 \pm 34$ & $0.4 \pm 0.8$ & $7.8 \pm 18.7$ & $69 \pm 68$ & $1.3 \pm 1.4$ \\
\hline Dolichopodidae & $\operatorname{Pr}$ & $0.4 \pm 1.0$ & $6 \pm 14$ & $0.6 \pm 1.9$ & - & - & - \\
\hline Muscidae & $\operatorname{Pr}$ & - & - & - & $0.1 \pm 0.2$ & $4 \pm 9$ & $0.1 \pm 0.3$ \\
\hline Tipulidae & $\operatorname{Pr}$ & $1.8 \pm 4.9$ & $17 \pm 39$ & $1.4 \pm 3.2$ & - & - & - \\
\hline Collembola & $\mathrm{Co}-\mathrm{Ga}$ & - & - & - & $0.1 \pm 0.3$ & $4 \pm 13$ & $0.1 \pm 0.2$ \\
\hline Hydracarina & $\operatorname{Pr}$ & $* *$ & $2 \pm 6$ & $* *$ & $0.3 \pm 0.7$ & $17 \pm 45$ & $0.1 \pm 0.3$ \\
\hline Shannon-Wiener diversity & & & 2.50 & & & 2.56 & \\
\hline Pielou's evenness & & & 0.69 & & & 0.67 & \\
\hline
\end{tabular}

$\mathrm{A}=$ abundance, $\mathrm{D}=$ density, $\mathrm{B}=$ biomass. FFG = Functional Feeding Groups, Co-Ga = collector-gatherers, Co-Fil = collector-filterers, $\operatorname{Pr}=$ predators, $\mathrm{Shr}=$ shredders, $\mathrm{Scr}=$ scrapers. $\left({ }^{*}\right)=$ undefined, $(* *)=$ value $<0.1,(-)=$ absent. 
exclusively from the lotic phase (Hirudinea, Tipulidae, Dolichopodidae, Leptohyphidae and Leptophlebiidae) and $26 \%$ from the semi-lentic phase (Coelotanypus, Fittkauimyia, Pentaneura, Dicrotendipes, Stenochironomus, Muscidae, Coenagrionidae, Hydropsychidae, Leptoceridae, Odontoceridae, Polycentropodidae, Veliidae and Collembola).

\section{Discussion}

Lower electric conductivity, turbidity, and nutrients and increased water temperature were possible consequences of the spillways and Amador Aguiar I Dam operations because the higher precipitation in 2008 would have been expected to have had the opposite effects. Generally, dams act as settling basins, increasing water transparency by retention of fine sediments (Kondolf, 1997). Amador Aguiar I Dam, together with the upstream reservoirs (Nova Ponte and Miranda), have probably incorporated most phosphorus and nitrogen and released better quality water downstream. This pattern was initially observed in a sequence of large reservoirs in the Tietê River (SP - Southeastern Brazil) (Barbosa et al., 1999), later confirmed for the Paraná River (PR - Southern Brazil) (Roberto et al., 2009), but not necessarily for the lower São Francisco River (MG Southeastern Brazil) (Callisto et al., 2005). As water passes through lentic ecosystems its upper layers heat; release of this layer warms downstream reaches. After water is released from dams its temperature tends to balance with the prevailing air temperature (Lessard and Hayes, 2003). The highest air temperature recorded during the semi-lentic phase was $28.3 \pm 3.4{ }^{\circ} \mathrm{C}$, vs. $26.9 \pm 2.0^{\circ} \mathrm{C}$ for the lotic phase, which contributed to water warming. The physical and chemical quality of the water remained within the limits established by Brazilian laws for human consumption (total-P $0.1 \mathrm{mg} / \mathrm{L}$; total-N $3.7 \mathrm{mg} / \mathrm{L}$; dissolved oxygen over $6.0 \mathrm{mg} / \mathrm{L}, \mathrm{pH} 6.0$ to 9.0 ; turbidity $40.0 \mathrm{NTU}$ ) (Brasil, 2005).

However, approaches based exclusively on physical and chemical measures do not reflect the condition of aquatic ecosystems (Karr and Dudley, 1981). Research conducted in the Cotter River in Australia, which is regulated by large hydroelectric dams, confirmed that monitoring abiotic variables was insufficient to indicate the impact of river habitat fragmentation. However, after including data about the structure of benthic macroinvertebrate communities, the negative effects became evident (Nichols et al., 2006). Aquatic fauna require specific habitat structure and flow regime conditions that are independent of water quality (Hannaford et al., 1997).

Declines in taxonomic diversity are commonly observed in benthic macroinvertebrate communities in reduced flow reaches (Cazaubon and Giudicelli, 1999; McIntosh et al., 2002; Kinzie et al., 2006). The decrease in water volume results in habitat loss and a decrease in the quantity and quality of food, leading to the disappearance of some taxa (McKay and King, 2006). This pattern, however, was not observed in this study. Because the response of benthic macrofauna during a disturbance depends on their resistance or susceptibility to the stressor agents, it is probable that changes in river habitats during the semilentic phase were of low impact to the local community, which was previously dominated by generalist groups (e.g., Chironomidae, Ceratopogonidae and Oligochaeta). These taxa are classified as tolerant to human modifications (Ogbeibu and Oribhabor, 2002; Brendenhand and Samways, 2009). According to Dewson et al. (2007c), the initial composition of communities controls the magnitude and direction of changes resulting from reduced flows. They emphasized that despite several species of macroinvertebrates being limited by flow, others are generalists less affected by flow alterations and therefore thrive in environments with variable hydraulic conditions. The presence of alien mollusks (Corbicula fluminea and Melanoides tuberculatus) during the lotic phase suggests that the benthic macroinvertebrate communities were already altered prior to flow reduction and spillway construction.

Since the 1990s, two large hydroelectric dams (Miranda and Nova Ponte) have operated upstream of the studied reach (Rodrigues, 2002) and their negative effects probably have spread downstream, as has been reported in the regulated Paraná river, Brazil (Stevaux et al., 2009). When rivers lose their natural flow regimes, they become homogenous (Moyle and Mount, 2007) and typically support a less diverse fauna (Poff et al., 2007). In undammed rivers, flood and drought pulses often act as selective forces, eliminating alien invaders not adapted to such conditions (Lytle and Poff, 2004). Therefore, by reducing peak flows, increasing low flows and creating semi-lentic reservoirs, dams may facilitate bioinvasion (Bunn and Arthington, 2002).

Although the categorization of benthic macroinvertebrates into functional feeding groups constitute a valuable tool for understanding food web relationships or for elaborating predictions in impact assessment studies (Vallania and Corigliano, 2007), the results obtained in this study disagreed with the proposed predictions. The collector-gatherers, feeding on fine fragmented organic matter deposited in the sediment (Wallace and Webster, 1996), were numerically dominant during the lotic phase, when the stretch of river rapids were well developed. On the other hand, the collector-filterers, feeding on drifting particles (Wallace and Merritt, 1980), dominated the community during the semi-lentic phase, when pools and backwaters were the major habitats and sources of phytoplankton production.

It is noteworthy that feeding habits of macroinvertebrates are not yet clearly defined (Motta and Uieda, 2004). During laboratory experiments, it was confirmed that some Chironomidae, typically non-scrapers, can use riparian vegetation leaf debris as a complementary food source (Callisto et al., 2007). Stomach analysis also indicated that various organisms traditionally characterised as predators in one geographical area, can feed as collectors at other sites (Silva et al., 2008). Food chains in most neotropical aquatic ecosystems are dominated by generalist feeders, with opportunistic behaviours aiding their occupation of multiple trophic positions (Tomanova et al., 2006). Filterers, for example, are not restricted to feeding on fine 
particulate organic matter that collects in their nets. They also capture food by direct interception or electrostatic attraction, which are important strategies in low flow conditions (Wallace and Merritt, 1980).

Macroinvertebrates exhibited higher density during the semi-lentic phase. Increased densities occur when reduced flows concentrate individuals in residual pools (Cortes et al., 2002; Dewson et al., 2007c). However, in the semi-lentic phase, the spillways restored water volumes to levels similar to those in the lotic phase. Thus, the increased total density was unlikely a result of reduced habitat availability. Two genera of Chironomidae (Ablabesmyia and Tanytarsus) and two families of Trichoptera (Leptoceridae and Polycentropodidae) increased densities in the semi-lentic phase. Ablabesmyia feeds on Chironomidae larvae and other smaller macroinvertebrates (Epler, 2001; Callisto et al., $2002 b$ ). Considering that the abundance of predators depends directly on prey availability (Vannote et al., 1980), the increase in the total number of macroinvertebrates and Chironomidae may have contributed to the increased numerical density of this genus. Tanytarsus inhabits sand substrates and is commonly found in environments with low water flux (Roque et al., 2004; Takahashi et al., 2008). The Leptoceridae and Polycentropodidae also include taxa that inhabit ponds and backwaters (Pérez, 1988; MartinsSilva et al., 2008). Their increased densities probably resulted from additional semi-lentic habitats.

Total biomass did not vary significantly between the two phases and was predominantly represented by the alien bivalve Corbicula fluminea. This is a filtering species, and its biomass consists basically of carbon from phytoplankton (Vaughn and Hakenkamp, 2001). This species lacked natural enemies (e.g., parasites and predators) in the invaded areas and can filter huge amounts of water (Sousa et al., 2008), which probably explains its high biomass even in an environment with limited productivity.

Significant changes in faunal composition occurred following flow reduction and formation of semi-lentic habitats; however there was overlapping of communities (ANOSIM R value $=0.3$ ). In dammed environments, the replacement of river species by lentic species may take several years (Voshell and Simmons, 1984). When studying the succession of benthic macroinvertebrates in a newly formed lentic environment, Bass (1992) noted that the initial colonisers were species that already inhabited the river before the dam and were resilient to the new hydraulic situation. Afterwards, species arrived from adjacent areas probably by dissemination from winged adults. At the end of three years, the author observed that $80 \%$ of the original taxa disappeared and new ones were established in the area. Published data suggests that a period ranging from 20 to 36 months is needed for new communities of macroinvertebrates to stabilise in a newly formed lentic system (Solomini et al., 2003; Williams et al., 2008). During research downstream of Flaming George Dam (USA), Vinson (2001) observed small changes in the invertebrate fauna during the first years after damming: large changes were detected five years after damming.
Another possible explanation for this pattern (overlapped communities) could be derived from the taxonomic resolution used in this study (mainly Family). According to Bailey et al. (2001) the responses of aquatic communities to environmental gradients are adequately discriminated when organisms are identified to species; higher taxonomic levels seem to be disadvantageous in detecting transitional impairments. This is an important aspect to be considered; however, species-level identification in Brazil is difficult because of insufficient taxonomic knowledge and lack of comprehensive taxonomic keys (Buss and Vitorino, 2010). Family identification appears to be good for assessing variation in benthic macroinvertebrate community structure, particularly in impact assessment studies (Melo, 2005; Corbi and Trivinho-Strixino, 2006). Moreover, Bowman and Bailey (1997) also suggested that multivariate characterizations of community composition are not strongly affected by taxonomic resolution. They analyzed ten data sets from published studies of freshwater benthic macroinvertebrates and concluded that genus-level identification did not usually add distinct information to the description of community patterns than higher levels (e.g, Family).

Communities colonising homogeneous habitats with little environmental variation tend to be fragile and susceptible to physical disturbance (Armitage, 2006). One way to minimise these problems would be to establish a flow regime in the reach that reflects the hydrometeorological variations of the basin, and is not confined to a constant minimal value of $7 \mathrm{~m}^{3} . \mathrm{s}^{-1}$. In this study, the sampling regime was similar to conventional methods (the sediment samples were collected along six random sites in the same reach and used as replicas because there is no other regulated river reach with similar characteristics in Brazil). Moreover, the sampling period was relatively short (1 year/phase), which may be difficult to reveal seasonal variations - atypical years. Therefore, the results obtained should be interpreted carefully and critically, considering these shortcomings. Currently, predicting how artificially flows affect lotic biota and assessing how much water a regulated river reach below a dam needs to preserve its ecological sustainability have been a challenging task for many water managers (Bunn and Arthington, 2002). In fact, they have properly applied instream flow incremental methodology (IFMI) for some fish groups (e.g. salmonids) (Dewson et al., 2007a), but its development and use for benthic macroinvertebrates have still been limited by restrictions in collection of adequate samples (large numbers), accurately taxonomic identification of species and construction of habitat suitability curves (Gore et al., 2001).

In summary, significant changes in taxonomic composition and total density of macroinvertebrates were detected after flow reduction and spillway construction. Statistically differences in richness, total biomass, and diversity were not observed. The macroinvertebrate assemblages were characterised by the numerical dominance of tolerant organisms and by high biomasses of an alien species, suggesting that this reach of the Araguari River was already impaired before its conversion to semi-lentic 
conditions. Since the early 1990s, the Araguari River basin has experienced dam construction and the replacement of native vegetation by pasture and crops, leading to altered biological communities and have altered ecosystem function. Because the restructuring of benthic fauna in dammed rivers is gradual, a periodic biomonitoring programme of this regulated reach is needed to assess the long-term biotic effects of altered flows.

Acknowledgements - We thank Consórcio Capim Branco Energia for project funding; CNPq for a Master's scholarship to DMM and a research fellowship to MC; US Fish \& Wildlife Service, CAPES and FAPEMIG for supporting the development of this study; Rener Gregório for field work assistance; colleagues of the Laboratório de Ecologia de Bentos - UFMG for sample processing; Daniel Coscarelli, Laboratório de Malacologia - UFMG, for identifying Corbicula fluminea, Prof. Gilmar B. Santos - PUC/MG, Prof. José F. Bezerra-Neto - UFMG, and Prof. José Francisco Gonçalves-Júnior - UNB for numerous suggestions and Robert M. Hughes for review and English editing. This paper was written while MC was a sabbatical visitor (CAPES fellowship No. 4959/09-4) at the IMAR, Universidade de Coimbra, Portugal.

\section{References}

AGOSTINHO, AA., THOMAZ, SM. and GOMES, LC., 2005. Conservação da biodiversidade em águas continentais do Brasil. Megadiversidade, vol. 1, no. 1, p. 70-78.

AGOSTINHO, AA., PELICICE, FM. and GOMES, LC., 2008. Dams and the fish fauna of the Neotropical region: impacts and management related to diversity and fisheries. Brazilian Journal of Biology, vol. 68, no. 4 (suppl.), p. 1119-1132.

ALMEIDA, EF., OLIVEIRA, RB., MUGNAI, R., NESSIMIAN, JL. and BAPTISITA, DF., 2009. Effects of small dams on the benthic community of streams in an Atlantic Forest area of Southeastern Brazil. International Review of Hydrobiology, vol. 94, no. 2, p. 179-193. http://dx.doi.org/10.1002/iroh.200811113

American Public Health Association - APHA, 1992. Standard Methods for the Examination of Water and Wastewater. Washington: American Public Health Association.

ARMITAGE, PD., 2006. Long-term faunal changes in a regulated and an unregulated stream - Cow Green thirty years on. River Research and Applications, vol. 22, no. 9, p. 947-966. http:// dx.doi.org/10.1002/rra.952

BAILEY, RC., NORRIS, RH. and REYNOLDSON, TB., 2001. Taxonomic resolution of benthic macroinvertebrate communities in bioassessments. Journal North American Benthological Society, vol. 20, no. 2, p. 280-286. http://dx.doi.org/10.1899/09-095.1

BARBOSA, FAR., PADISÁK, J., ESPÍNDOLA, ELG., BORICS, G. and ROCHA, O., 1999. The cascading reservoir continuum concept (CRCC) and its applications to the river Tietê-basin, São Paulo State, Brazil. In TUNDISI, JG. and STRASKABA, M. Theoretical Reservoir Ecology and its Applications. Leiden: Backhuys Publishers. p. 425-438.

BASS, D., 1992. Colonization and succession of benthic macroinvertebrates in Arcadia Lake, a South-Central USA reservoir. Hydrobiologia, vol. 242, no. 2, p.122-131.

BOWMAN, MF. and BAILEY, RC., 1997. Does taxonomic resolution affect the multivariate description of the structure of freshwater benthic communities? Canadian Journal of Fisheries and Aquatic Sciences, vol. 54, no. 8, p. 1802-1807.

Brasil. Resolução no 357 de 17 de março de 2005 do Conselho Nacional do Meio Ambiente. Diário Oficial da União. Secção 1, $\mathrm{n}^{\circ}$ 53, 18 de março de 2005. p. 58-63. Available from: <http:// www.mma.gov.br/port/conama/res/res05/res35705.pdf >. Access in: 22 abr. 2009.

BRENDENHAND, E. and SAMWAYS, MJ., 2009. Impact of a dam on benthic macroinvertebrates in a small river in a biodiversity hotspot: Cape Floristic Region, South Africa. Journal of Insect Conservation, vol. 13, no. 3, p. 297-307. http://dx.doi.org/10.1007/ s10841-008-9173-2

BRITTAIN, JE. and L'ABÉE-LUND, JH., 1995. The environmental effect of dams and strategies for reducing their impact. In SANTBEGGEN, L. and WESTEN, CJV. Reservoirs in River Basin Development. Rotterdam: AA Balkema. p. 129-138.

BUNN, SE. and ARTHINGTON, AH., 2002. Basic principles and ecological consequences of altered flow regimes for aquatic biodiversity. Environmental Management, vol. 30, no. 4, p. 492507. http://dx.doi.org/10.1007/s00267-002-2737-0

BUSS, DF. and VITORINO, AS., 2010. Rapid Bioassessment Protocols using benthic macroinvertebrates in Brazil: evaluation of taxonomic sufficiency. Journal North American Benthological Society, vol. 29, no. 2, p. 562-571. http://dx.doi.org/10.1899/09-095.1

CAllisto, M. and ESTEVES, FA., 1996. Composição granulométrica do sedimento de um lago amazônico impactado por rejeito de bauxita e um lago natural. Acta Limnologica Brasiliensia, vol. 8, p. 115-126.

CALLISTO, M., FERREIRA, W., MORENO, P., GOULART, M. and PETRUCIO, M., 2002a. Aplicação de um protocolo de avaliação rápida da diversidade de habitats em atividades de ensino e pesquisa (MG-RJ). Acta Limnologica Brasiliensia, vol. 14 , no. 1, p. 91-98.

CALLISTO, M., GONÇALVES-JUNIOR, JF. and GRAÇA, MS., 2007. Leaf litter as possible food source for chironomids (Diptera) in Brazilian and Portuguese streams. Revista Brasileira de Zoologia, vol. 24, no. 2, p. 442-448.

CALLISTO, M., GOULART, M., BARBOSA, FAR. and ROCHA, O., 2005. Biodiversity assessment of benthic macroinvertebrate along a reservoir cascade in the lower São Francisco river (Northeastern Brazil). Brazilian Journal of Biology, vol. 65, no. 2, p. 1-6. http://dx.doi.org/10.1590/S1519-69842005000200006

CALLISTO, M., MORENO, P., GONÇAVELS-JUNIOR, JR., LEAL, JJF. and ESTEVES, FA., 2002b. Diversity and biomass of Chironomidae (Diptera) larvae in an impacted coastal lagoon in Rio de Janeiro, Brazil. Brazilian Journal of Biology, vol. 62, no. 1 , p. $77-84$

CAZAUBON, A. and GIUDICELLI, J., 1999. Impact of the residual flow on the physical characteristics and benthic community (algae, invertebrates) of a regulated Mediterranean river: the Durance, France. Regulated Rivers, vol. 15, no. 5, p. 441-461. http:// dx.doi.org/10.1002/(SICI)1099-1646(199909/10)15:5<441::AIDRRR558>3.0.CO;2-9

Consórcio Capim Branco Energi - CCBE, 2007. Soleiras vertentes - obras concluídas. Marra: Araguari. 4 p. Informativo.

CLARKE, KR. and GREEN, RH., 1988. Statistical design and analysis for a "biological effects" study. Marine Ecology Progress Series, vol. 46, no. 1-3, p. 213-226. 
CORBI, JJ. and TRIVINHO-STRIXINO, S., 2006. Influence of taxonomic resolution of stream macroinvertebrate communities on the evaluation of different land uses. Acta Limnologica Brasiliensia, vol. 18, no. 4, p. 469-475.

CORTES, RMV., FERREIRA, MT., OLIVEIRA, SV. and OLIVEIRA, D., 2002. Macroinvertebrate community structure in a regulated river segment with different flow conditions. River Research and Applications, vol. 18, no. 4, p. 367-382.

COSTA, C., IDE., S. and SOMONKA, CES., 2006. Insetos Imaturos: metaformose e identificação. Ribeirão Preto: Holos. 249 p.

CUMMINS, KW., MERRITT, TRW. and ANDRADE, PCN., 2005. The use of invertebrate functional groups to characterize ecosystem attributes in selected streams and rivers in southeast Brazil. Studies on Neotropical Fauna and Environment, vol. 40, no. 1, p. 71-90.

DEWSON, ZS., JAMES, ABW. and DEATH, RG., 2007a. A review of the consequences of decreased flow for instream habitat and macroinvertebrates. Journal North American Benthological Society, vol. 26, no. 3, p. 401-41. http://dx.doi.org/10.1899/06-110.1

-, 2007b. Invertebrate responses to short-term water abstraction in small New Zeland streams. Freshwater Biology, vol. 52, no. 2 , p. 357-369.

-, 2007c. Invertebrate community responses to experimentally reduced discharge in small streams of different water quality. Journal North American Benthological Society, vol. 26, no. 4, p. 754-766.

EPLER, JH., 2001. Indentification manual for the larval Chironomidae (Diptera) of North and South Carolina. Raleigh: North Carolina Department of Environment and Natural Resources Division of Water Quality. 495 p.

FERNÁNDEZ, HR. and DOMÍNGUEZ, E., 2001. Guía para la determinación de los artrópodos bentónicos sudamericanos. Tucuman: Universidad Nacional de Tucuman. 282 p.

FJELLHEIM, A. and RADDUM, GG., 1996. Weir building in a regulated west Norwegian river: long-term dynamics of invertebrates and fish. Regulated Rivers, vol. 12, no. 4-5, p. 501-508. http:// dx.doi.org/10.1002/(SICI)1099-1646(199607)12:4/5<501::AIDRRR414>3.0.CO;2-F

GEORGIAN, T. and THORP, JH., 1992. Effects of microhabitat selection on feeding rates of net-spinning caddisfly larvae. Ecology, vol. 73, no. 1, p. 229-240. http://dx.doi.org/10.2307/1938734

GORE, JA., LAYZER, JB. and MEAD, J., 2001. Macroinvertebrate instream flow studies after 20 years: a role in stream management and restoration. Regulated Rivers, vol. 17, no. 4-5, p. 527-542.

GRAÇA, MAS., 2001., The role of invertebrates on leaf litter decomposition in stream - a review. International Review of Hydrobiology, vol. 86, no. 4-5, p. 383-393.

HANNAFORD, MJ., BARBOUR, MT. and RESH, VW., 1997. Training reduces observer variability in visual - based assessments of stream habitat. Journal North American Benthological Society, vol. 16 , no. 4 , p. $853-860$

HANSEN, RA., HART, DD. and MERZ, RA., 1991. Flow mediates predator-prey interactions between triclad flatworms and larval black flies. Oikos, vol. 60, p. 187-196. http://dx.doi. org/10.2307/3544865

HARGROVE, WW. and PICKERING, J., 1992. Pseudoreplication: a sine qua non for regional ecology. Landscape Ecology, vol. 6, no. 4 , p. 251-258.
HURLBERT, SH., 1984. Pseudoreplication and the design of ecological field experiments. Ecological Monographs, vol. 54, no. 2, p. 187-211. http://dx.doi.org/10.2307/1942661

JAMES, ABW., DEWSON, ZS. and DEATH, RG., 2007. The effect of experimental flow reductions on macroinvertebrate drift in natural and stream side channels. River Research and Applications, vol. 24 , no. 1, p. 22-35. http://dx.doi.org/10.1002/rra.1052

KARR, JR. and DUDLEY, DR., 1981. Ecological perspective on water quality goals. Environmental Management, vol. 5, no. 1 , p. 55-68.

KINZIE, RAI., CHONG, C., DEVRELL, J., LINDSTROM, D. And WOLF, R., 2006. Effects of water removal on a Hawaiian stream ecosystem. Pacific Scientific, vol. 60, no. 1, p. 1-47.

KONDOLF, GM., 1997. Hungry water: effects of dams and gravel mining on river channels. Environmental Management, vol. 21 , no. 4 , p. $533-551$

LESSARD, JL. and HAYES, DB., 2003. Effects of elevated water temperature on fish and macroinvertebrate communities below small dams. River Research and Applications, vol. 19, no. 7, p. 721-732.

LYTLE, DA. and POFF, NL., 2004. Adaptation to natural flow regimes. Trends in Ecology and Evolution, vol. 19, no. 2, p. 94-100.

MAGURRAN, AE., 2004. Measuring biological diversity. Oxford: Blackwell Science. $260 \mathrm{p}$

MALMQVIST, B. and SACKMANN, G., 1996. Changing risk of predation for a filter-feeding insect along a current velocity gradient. Oecologia, vol. 108, no. 3, p. 450-458. http://dx.doi. org/10.1007/BF00333721

MARTINS-SILVA, MJ., ENGEL, DW., ROCHA, FM. and ROCHA, FM., 2008. Imaturos de Trichoptera na bacia do rio Paraná, GO, com novos registros de gênero. Neotropical Entomology, vol. 37, no. 6, p. 735-738. PMid:19169565

McINTOSH, MD., BENBOW, ME. and BURKY, AJ., 2002. Effects of stream diversion on riffle macroinvertebrate communities in a Maui, Hawaii, stream. River Research and Applications, vol. 18, no. 6 , p. 569-581.

McKAY, SF. and KING, AJ., 2006. Potential ecological effects of water extraction in small, unregulated streams. River Research and Applications, vol. 22, no. 9, p. 1023-1037.

MELO, AS., 2005. Effects of taxonomic and numeric resolution on the ability to detect ecological patterns at local scale using stream macroinvertebrates. Archiv für Hydrobiologie, vol. 164, no. 3 , p. 309-323.

MERRITT, RW. and CUMMINS, KW., 1996. An introduction to the aquatic insects of North America. Iowa: Kendall Hunt. 862 p.

MICHELLAND, RJ., COMBES, S., MONTEILS, V., CAUQUIL, L., GIDENNE, T. and FORTUN-LAMOTHE, L., 2010. Molecular analysis of the bacterial community in digestive tract of rabbit. Anaerobe, vol. 16, no. 2, p. 61-65. PMid:19460451

MOTTA, RL. and UIEDA, VS., 2004. Diet and trophic groups of an aquatic insect community in a tropical stream. Brazilian Journal of Biology, vol. 64, no. 4, p. 809-817. http://dx.doi. org/10.1590/S1519-69842004000500010

MOYLE, PB. and MOUNT, JF., 2007. Homogeneous rivers, homogeneous faunas. Proceedings of the National Academy of Sciences USA, vol. 104, no. 14, p. 5711-5712. PMid: 15744421 
NICHOLS, S., NORRIS, R., MAHER, W. and THOMS, M., 2006. Ecological effects of serial impoundment on the Cotter River, Australia. Hydrobiologia, vol. 572, no. 1, p. 255-273. http:// dx.doi.org/10.1007/s10750-005-0995-6

NILSSON, C. REIDY, CA., DYNESIUS, M. and REVENGA, C., 2005. Fragmentation and flow regulation of the world's large river systems. Science, vol. 308, no. 5720, p. 405-408.

OGBEIBU, AE. and ORIBHABOR, BJ., 2002. Ecological impact of river impoundment using benthic macro-invertebrates as indicators. Water Research, vol. 36, no. 10, p. 2427-2436. PMid:12153008. http://dx.doi.org/10.1016/S0043-1354(01)00489-4

PÉREZ, GH., 1988. Guía para el estudio de los macroinvertebrados acuáticos del Departamento de Antioquia. Bogotá: Universidad de Antioquia. 217 p.

POFF, NL., OLDEN, JD., MERRITT, DM. and PEPIM, DM., 2007. Homogenization of regional river dynamics by dams and global biodiversity implications. Proceedings of the National Academy of Sciences USA, vol. 104, no. 14, p. 5732-5737.

ROBERTO, MC., SANTANA, NF. and THOMAZ, SM., 2009. Limnology in the Upper Paraná River floodplaIn large-scale spatial and temporal patterns, and the influence of reservoirs. Brazilian Journal of Biology, vol. 69, no. 2 (supl.), p. 717-725.

RODRIGUES, SC., 2002. Mudanças ambientais na região do Cerrado: Análise das causas e efeitos da ocupação do solo sobre o relevo: O caso da bacia hidrográfica do rio Araguari - MG. Geousp, no. 12, p. 105-124.

ROQUE, FO. and TRIVINHO-STRIXINO, S., 2008. Four new species of Endotribelos Grodhaus, a common fallen fruit-dwelling chironomid genus in Braziliam streams (Diptera: Chironomidae: Chironominae). Studies on Neotropical Fauna and Environment, vol. 43, no. 3, p. 191-207.

ROQUE, FO., CORREIA, LCS., TRIVINHO-STRIXINO, S. and STRIXINO, G., 2004. A review of Chironomidae studies in lentic system in the state of São Paulo, Brazil. Biota Neotropica, vol. 4, no. 2, p. 1-19.

ROSA, R., BRITO, JLS. and LIMA, SC., 2006. Uso do solo e cobertura vegetal na área de influência do AHE Capim Branco I. Sociedade e Natureza, vol. 18, no. 34, p. 133-150.

SILVA, FL., MOREIRA, DC., BOCHINI, GL. and RUIZ, SS., 2008. Hábitos alimentares de larvas de Chironomidae (Insecta: Diptera) do córrego Vargem Limpa, Bauru, SP, Brasil. Biotemas, vol. 21 , no. 2, p. 155-159.

Sistema de Metereologia e Recursos Hídricos de Minas Gerais - SIMGE, 2010. Mapas Climáticos Mensais. Available from: $<$ http://www.simge.mg.gov.br>. Access in: 22 mar. 2010.

SOLOMINI, AG., RUGGIERO, A., BERNARDINI, V. and CARCHINI, G., 2003. Temporal pattern of macroinvertebrate diversity and production in a new man made shallow lake. Hydrobiologia, vol. 506-509, no. 1-3, p. 373-379.

SOUSA, R., ANTUNES, C. and GUILHERMINO, L., 2008. Ecology of the invasive Asian clam Corbicula fluminea (Müller, 1774) in aquatic ecosystem: an overview. Annales de Limnologie International Journal of Limnology, vol. 44, no. 2, p. 85-94. http://dx.doi.org/10.1051/limn:2008017

STEVAUX, JC., MARTINS, DP. and MEURER, M., 2009. Changes in a large regulated tropical river: the Paraná river downstream from Porto Primavera dam, Brazil. Geomorphology, vol. 113 , no. $3-4$, p. $230-238$.
TAKAHASHI, MA., HIGUTI, J., BAGATINI, YM., ZVIEJKOVSKI, IP. and VELHO, LMF., 2008. Composition and biomass of larval chironomid (Insecta, Diptera) as potential indicator of trophic conditions in southern Brazil reservoirs. Acta Limnologica Brasiliensia, vol. 20, no. 1, p. 5-13.

TOMANOVA, S., GOITIA, E. and HELESIC, J., 2006. Trophic levels and functional feeding groups of macroinvertebrates in Neotropical streams. Hydrobiologia, vol. 556, no.1, p. 221-265.

TRIVINHO-STRIXINO, S. and STRIXINO, G., 1995. Larvas de Chironomidae (Diptera) do estado de São Paulo: guia de identificação e diagnose dos gêneros. São Carlos: PPG-ERN/ UFSCAR. 229 p.

TUNDISI, JG. and MATSUMURA-TUNDISI, T., 2008. Limnologia. São Paulo: Oficina de Textos. 632 p.

TUNDISI, JG., MATSUMURA-TUNDISI, T. and TUNDISI, JEM., 2008. Reservoirs and human well-being: new challenges for evaluating impacts and benefits in the neotropics. Brazilian Journal of Biology, vol. 68, no. 4 (supl.), p. 1113-1135.

VALADARES, CF., 2007. Alterações nas características limnológicas e na comunidade zooplanctonica do rio Araguari (MG) em função as obras hidraúlicas da UHE Capim Branco I. Belo Horizonte: Universidade Federal de Minas Gerais. 130 p. Dissertação de Mestrado em Conservação e manejo da vida silvestre.

VALLANIA, A. and CORIGLIANO, MDC., 2007. The effect of regulation caused by a dam on the distribution of the functional feeding groups of the benthos in the sub basin of the Grande River (San Luis, Argentina). Environmental Monitoring and Assessment, vol. 124, no. 1-3, p. 201-209. PMid:17160442. http:// dx.doi.org/10.1007/s10661-006-9218-5

VANNOTE, RL., MINSHALL, GW., CUMMINS, KW., SEDELL, JR. and CUSHING, CE., 1980. The river continuum concept. Canadian Journal of Fisheries and Aquatic Sciences, vol. 37, no. 1, p. 130-137.

VAUGHN, CC. and HAKENKAMP, CC., 2001. The functional role of burrowing bivalves in freshwater ecosystems. Freshwater Biology, vol. 46, no. 1, p. 1431-1446.

VINSON, MR., 2001. Long-term dynamics of an invertebrate assemblage downstream from a large dam. Ecological Application, vol. 11, no. 3, p. 711-730.

VOSHELL, JR. and SIMMONS, GM., 1984. Colonization and succession of benthic macroinvertebrates in a new reservoir. Hydrobiologia, vol. 112, no. 1, p. 27-39.

WALLACE, JB. and MERRITT, RW., 1980. Filter-feeding ecology of aquatic insects. Annual Review of Entomology, vol. 25, p. 103132. http://dx.doi.org/10.1146/annurev.en.25.010180.000535

WALLACE, JB. and WEBSTER, JR., 1996. The role of macroinvertebrates in stream ecosystem function. Annual Review of Entomology, vol. 41, p. 115-139. PMid:15012327. http://dx.doi. org/10.1146/annurev.en.41.010196.000555

WILLIAMS, P., WHITFIELD, M. and BIGGS, J., 2008. How can we make new ponds biodiverse? A case study monitored over 7 years. Hydrobiologia, vol. 597, p. 137-148. http://dx.doi. org/10.1007/s10750-007-9224-9

ZAR, JH., 1996. Biostatistical analysis. New Jersey: PrenticeHall. 662 p. 
